\title{
Video Article \\ Operating Procedures of the Electrochemotherapy for Treatment of Tumor in Dogs and Cats
}

\author{
Natasa Tozon ${ }^{1}$, Ursa Lampreht Tratar $^{2}$, Katarina Znidar ${ }^{3}$, Gregor Sersa ${ }^{2}$, Justin Teissie ${ }^{4,5}$, Maja Cemazar ${ }^{2,3}$ \\ ${ }^{1}$ Clinic for Surgery and Small Animals, Veterinary Faculty, University of Ljubljana \\ ${ }^{2}$ Department of Experimental Oncology, Institute of Oncology Ljubljana \\ ${ }^{3}$ Faculty of Health Sciences, University of Primorska \\ ${ }^{4}$ IPBS (Institut de Pharmacologie et de Biologie Structurale), CNRS \\ ${ }^{5}$ IPBS (Institut de Pharmacologie et de Biologie Structurale), Université de Toulouse
}

Correspondence to: Maja Cemazar at mcemazar@onko-i.si

URL: https://www.jove.com/video/54760

DOI: doi: $10.3791 / 54760$

Keywords: Cancer Research, Issue 116, electrochemotherapy, operating procedure, veterinary oncology, mast cell tumor, treatment, dog

Date Published: 10/24/2016

Citation: Tozon, N., Lampreht Tratar, U., Znidar, K., Sersa, G., Teissie, J., Cemazar, M. Operating Procedures of the Electrochemotherapy for Treatment of Tumor in Dogs and Cats. J. Vis. Exp. (116), e54760, doi:10.3791/54760 (2016).

\section{Abstract}

Electrochemotherapy (ECT) is a local approach which is used for treating solid tumors of different histologies. Its mechanism is based on cell membrane permeabilization by means of "electroporation". To achieve the "electroporation" of the cells, electric pulses are generated by a generator and delivered to the target tissue by the use of electrodes. Electroporation is a physical method which is used to introduce molecules, like cytostatic drugs, into the cells that could not pass the cell membrane on their own. In electrochemotherapy, currently, cisplatin and bleomycin are clinically used. Electrochemotherapy antitumor effectiveness is high, for example up to $100 \%$ complete response of canine mast cell tumors smaller than $2 \mathrm{~cm}^{3}$ was achieved. Additionally, electrochemotherapy can be used for the treatment of inoperable tumors. One of the important characteristics of electrochemotherapy is that it can be effective as a one-time treatment only. However, in the case of failure or partial tumor response it can be repeated several times with equal or improved effectiveness. Electrochemotherapy is already a standard treatment for cutaneous and subcutaneous tumors of various histologies in human and veterinary oncology. Furthermore, several clinical studies exploiting electrochemotherapy for deep-seated tumors are on-going.

\section{Video Link}

The video component of this article can be found at https://www.jove.com/video/54760/

\section{Introduction}

ECT is a local ablative technique for the treatment of tumors of different histologies. Ablation results from the drug induced cell kill, which is a result of increased drug accumulation in the cells due to changed cell membrane permeability induced by direct delivery of electric pulses on the skin around the tumor after the intratumoral or intravenous injection of the drug. The main mechanism of ECT is to enhance the effectiveness of cytostatic drugs by increased intracellular drug accumulation, by cell membrane electropermeabilization (electroporation) ${ }^{1,2}$. Electroporation causes an increased drug uptake of two cytostatics: bleomycin and cisplatin, which are hydrophilic drugs with poor membrane permeability. Due to the increased cytotoxicity of bleomycin and cisplatin at the site of application of the electric pulse, low drug doses are needed for good antitumor effectiveness and consequently no or minimal systemic side effects of the drugs are observed ${ }^{3}$. Another characteristic of ECT is a tumor antivascular effect which results firstly in vascular lock, i.e., decreased blood flow within the tumor, and consequently the retention of cytostatics within it and secondly in a vascular disrupting action, i.e., direct killing of endothelial cells of small blood vessels leading to secondary tumor cell death ${ }^{4-6}$. The third mechanism of ECT antitumor effectiveness is the activation of the immune system as ECT elicits an immune response by tumor antigen shedding in the tumor surroundings. The involvement of the immune system is crucial for the complete eradication of the tumor?

ECT has shown great potential in human oncology, where ECT is already used as a standard treatment for cutaneous and subcutaneous tumors as it exhibits up to $80 \%$ of objective responses ${ }^{3}$. Additionally, ECT is used in several clinical studies treating tumor metastases in the brain $^{8}$, bone ${ }^{9}$ and liver ${ }^{10}$. Clinical studies in veterinary oncology have already demonstrated that ECT treatment results in up to $80 \%$ longlasting objective responses when treating cutaneous and subcutaneous tumors in dogs ${ }^{11,12}$, cats ${ }^{13}$ and horses ${ }^{14}$. The antitumor effectiveness is dependent on tumor size and tumors smaller than $2 \mathrm{~cm}^{3}$ respond better than bigger tumors ${ }^{15}$.

Also, a study comparing surgery with ECT in treating mast cell tumors in dogs showed that treatment with ECT resulted in $70 \%$ of complete responses while surgery resulted in $50 \%$ of complete responses ${ }^{11}$ demonstrating that ECT is an equivalent treatment to surgery. Additionally, ECT represents an alternative method for treating solid tumors to surgical treatment, specifically in those cases when owners do not consent to surgery or if the tumors are difficult to excise with clear margins mostly due to their location (e.g., infiltrating major vessels, tumors of the head or leg, in the anal region etc.). The aim of this paper is to define the operating procedures of ECT giving instructions for the use of cisplatin and 
bleomycin in combination with the application of electric pulses in order to safely and appropriately treat cutaneous and subcutaneous tumors in dogs and cats.

\section{Protocol}

This protocol was approved by the Ethical Committee of the Ministry of Agriculture, Forestry and Food and follows its guidelines. The protocol was validated on more than 80 dogs and 20 cats.

NOTE: ECT treatment consists of the administration of cisplatin or bleomycin and exposure of tumor nodules to electric pulses.

\section{Patient Selection}

NOTE: Patient selection allows the practitioner to determine whether the patient is suitable or not to benefit from this treatment.

1. Evaluate the history of the patient (general data about age, breed, sex and presence of any concurrent diseases, tumor type, evolution of the tumor with time, previous treatments and its results, recurrence and speed of recurrence, owner's expectations about the successfulness of the therapy).

2. Perform a physical examination of the patient

1. Evaluate the tumor in detail in order to make a possible clinical differential diagnosis and perform a fine needle aspirate for cytology evaluation and possible tissue biopsy for histology evaluation in order to determine the treatment area: if a potentially invasive tumor is presented treat the wide margins (make the decision about the size of the treated margins according to recommendations for any other treatment for the same type of tumor, e.g., mast cell tumor $2 r \leq 1 \mathrm{~cm}, 1 \mathrm{~cm}$ margin should be treated; $r$ - radius).

2. Evaluate the number and size of the nodules in order to prepare a treatment approach.

3. General examination

1. Implement a full general examination in order to make staging of the disease and check:

1. If there is no other concurrent disease which could precludes the treatment, if the patient has a history of any chronic infection (according to epidemiology in a certain area) or immune-mediated disease which could alter hematological or coagulation changes (perform the specific tests), If the animal is able to withstand a series of deep sedations and/or general anesthesia, If the patient has sensitivity to cisplatin (standard dosage is $60-70 \mathrm{mg} / \mathrm{m}^{2}$ body surface area / $21 \mathrm{~d}$ ) or bleomycin or has already received a cumulative dose of bleomycin exceeding $400.000 \mathrm{IU} / \mathrm{m}^{2}$.

2. Use diagnostic imaging techniques (radiography of the thorax and ultrasound examination of the abdomen) in order to check any possible metastases according to the tumor type.

\section{Laboratory Analysis}

1. Blood work

1. Perform complete hematology analyses with a biochemistry panel of blood urea nitrogen (BUN), creatinine, alanine-aminotransferase $(\mathrm{ALT})$, alkaline phosphatase (AF) and calcium to exclude advanced chronic renal and hepatic diseases and possible most frequent paraneoplastic syndromes in order to determine the clinical stage which may have an effect on the prognosis.

2. Do an additional specific blood analysis:

1. If the patient is given any medicine which could potentially alter changes in coagulation ability, check the coagulation profile.

2. If using invasive treatment (needle electrodes), check the platelet number; the number of platelets should be $>100$ or in case it is $<100$, the coagulation profile (PT, APTT, fibrinogen, D-dimer) should be normal.

3. In specific cases according to neoplastic disease and the clinical status of the patient check the total proteins, albumin, phosphors, sodium $(\mathrm{Na})$, potassium $(\mathrm{K})$, chloride $(\mathrm{Cl})$ and lipase concentration.

2. Tumor(s) histological analysis

1. Histology analysis is not necessary if the cytology diagnosis was performed previously. In the case of certain tumor types where the histological grade is a crucial prognostic factor (e.g., mast cell tumors) perform hematoxylin and eosin staining ${ }^{16}$.

NOTE: The biopsy (incisional or "punch") can be made before ECT during the same visit. In any case, it is good clinical practice to diagnose the tumor type.

NOTE: After the case is fully evaluated a thorough discussion and clarification with the animal's owner is needed. Explain to the owner what ECT is and what could be expected from ECT in terms of clinical results. Discuss with the owner the treatment course: the number of ECT sessions under general anesthesia or deep sedation, total duration of treatment, cost of treatment, possibilities of recurrence. Obtain the owner's written consent in the case of patient inclusion within a clinical trial of ECT, if the patient is actually eligible.

\section{Choice of Treatment Modality}

NOTE: For ECT in dogs use cisplatin and bleomycin. For ECT in cats use bleomycin only, as cisplatin is contraindicated in cats.

1. Treat each tumor separately. Determine the location and size of each nodule and the number of nodules to outline the treatment protocol. 
2. Calculate the volume of tumor nodules using the formula $V=a b^{2} \pi / 6$ ("a" is the larger diameter of the tumor nodule and " $b$ " the diameter of the tumor nodule perpendicular to "a") or $V=a b c \pi / 6$ ("a", "b" and "c" are three perpendicular diameters of the tumor nodule) .

\section{Choice of Operating Modality}

1. Selection of electrodes

NOTE: It is important to choose the optimal electrode type as electrode configuration affects the electrical field distribution in the tissue. Two types of electrode are used: plate and needle electrodes. Plate electrodes have parallel stainless steel plates: the inner distance between the electrodes depends on the size of the tumor and the capacity of the generator of electric pulses. Usually the distance between the electrodes is 6-8 $\mathrm{mm}$. Needle electrodes can be arranged in rows (parallel array) or in hexagonal geometry. The hexagonal electrode has a central needle and 6 needles in a circular array. The pulses are applied between the electrodes in the circular array and between them and the central one. The pulses are applied in both directions between each pair of electrodes. Choose the type of electrode according to the depth of the tumor nodule and provide appropriate electroporation in the whole desired treated area. Choose the electrodes according to the particular target tissue. In general, both types of electrodes can be used for the treatment.

2. Selection and preparation of cisplatin or bleomycin

NOTE: ECT consists of intratumoral administration of cisplatin or bleomycin or intravenous administration of bleomycin and exposure of tumor nodules to electric pulses.

1. Select an appropriate cytostatic and the route of its administration.

2. Dissolve cisplatin (cis-diamminedichloroplatinum II), if obtained as a powder, in distilled water at a concentration of $1 \mathrm{mg} / \mathrm{ml}$ (otherwise pharmaceutically prepared solutions could be used). Give cisplatin intratumorally.

3. Dissolve bleomycin in a physiological saline at a concentration of $3 \mathrm{mg} / \mathrm{ml}$ and give intratumorally or intravenously.

3. Anesthesia

NOTE: Depending on operating modality (Table 1) deep sedation or general anesthesia can be used.

1. Evaluate the patient according to The American Society of Anesthesiologists' (ASA) physical status classification and according to tumor type because of possible paraneoplastic syndromes.

4. Deep sedation

1. Use medetomidine $(0.08 \mathrm{mg} / \mathrm{kg})$ or double combination medetomidine $(0.025 \mathrm{mg} / \mathrm{kg})$ with butorphanol tartrate $(0.1 \mathrm{mg} / \mathrm{kg})$ in dogs or follow standard procedures for deep sedation in the specific hospital.

5. General anesthesia

1. Premedicate with acepromazine $(0.02 \mathrm{mg} / \mathrm{kg})$ and methadone $(2 \mathrm{mg} / \mathrm{kg})$.

2. Thirty min later induce general anesthesia with thiopental $(5 \mathrm{mg} / \mathrm{kg})$ or propofol $(1 \mathrm{mg} / \mathrm{kg})$.

3. Place endotracheal tube and maintain anesthesia with isoflurane in oxygen delivered via a T-piece anesthetic circuit.

4. During anesthesia use fluid support treatment with Hartmann's solution at a rate of $10 \mathrm{ml} / \mathrm{kg} / \mathrm{hr}$ and analgesia with a single dose of a non-steroid analgetic drug (e.g., carprofen $2-4 \mathrm{mg} / \mathrm{kg}$ ).

5. Use eye ointment to prevent dryness while under anesthesia. After treatment, supervise the patient until it regains sufficient consciousness to sternal recumbency. Keep the patient in the clinic for about 2 to $4 \mathrm{hr}$. NOTE: Standard procedures for general anesthesia in the specific hospital can also be followed.

6. Operating environment

NOTE: Personnel operating are trained ECT operators (DVM), anesthetists and assistants.

1. Use any ambulance room when ECT under deep sedation is performed or any surgical room where general anesthesia is allowed.

2. For sedation and/or general anesthesia use all required equipment according to the procedures for anesthesia.

3. For the ECT procedure see all required materials in the Materials List.

7. Operator and surrounding personnel protection

NOTE: Droplet exposure of the skin to antineoplastic drugs rarely causes acute problems. Cisplatin and bleomycin are not absorbed through intact skin. Cisplatin may cause only irritation. Cisplatin and bleomycin leakage could occur when injecting firm nodules or tumors. In case of needle stick injury, wash with abundance of water and take care of the wound according to the standard medical practice.

1. Protect the operator and surrounding assistants with masks and gloves. In case of skin exposure to cisplatin or bleomycin solution, rinse immediately and abundantly with water.

2. Set up biosafety measures according to a quality insurance program complying with local regulations about anticancer drug usage, if any.

8. Generators of electric pulses

NOTE: Applications of electric pulses can be carried out with the generator of electric pulses. Commonly, the apparatus is a square-wave pulse generator with integrated software and a touch-screen interface to select pre-designed electrical pulses. It is equipped with a hand-held electrode holder with a pulse-activating button attached to the generator by a cord. The delivery of electric pulses can be controlled by a foot switch. The generator of electric pulses should meet European Union standards: 2006/95/CE from 12/12/2006 concerning electronic material used within specific voltage limits, 2004/108/CE from 12/15/2004 concerning electromagnetic compatibility and should be compliant with the FDA's regulations.

9. Procedure of application of electric pulses

NOTE: The application of electric pulses follows the intratumoral administration of cisplatin or bleomycin, or intravenous administration of bleomycin. When administering intratumorally cytostatic should be applied slowly to prevent leakage. For intravenously administration cytostatics should be applied in a bolus lasting approximately $30 \mathrm{sec}$. A water-based gel is used to achieve good contact between the skin and the electrodes, either plate or needle, and to achieve less heterogeneous electric field distribution. The conductivity of gels should be between 0.5 to $1 \mathrm{~S} / \mathrm{m}^{17,18}$. The electric conductivity of the gel used was $0.5 \mathrm{~S} / \mathrm{m}$. The plate electrodes should be placed in such a way that the tumor is encompassed between them. Furthermore, to achieve better distribution of the electric field the application of electric pulses 
should be performed twice in a perpendicular direction. If the tumor is larger than the distance between the electrodes, the application of electrodes should start from the margins of the tumor (including the safety margin) and progress toward the center of the tumor. In the case of needle electrodes, care should be taken that all the needles are inserted into the tissue to achieve the appropriate distribution of the electric

field. The application of a gel is not needed. Otherwise, the procedure is the same as in the case of plate electrodes.

1. Depilate the skin in the area of application of electric pulses

2. Administer cisplatin or bleomycin. If cytostatic is administered intravenously wait for $8 \mathrm{~min}$ and the time interval for electric pulses delivery is $20 \mathrm{~min}$. If cytostatic is administered intratumorally start with the application of electric pulses immediately (within 1 min after injection).

3. Before application of electric pulses apply a water-based gel onto the depilated skin.

4. For plate electrodes use these pulse parameters: pulse duration $100 \mu \mathrm{sec}$, amplitude to electrode distance ratio $1,300 \mathrm{~V} / \mathrm{cm}$, frequency $1 \mathrm{~Hz}$ or $5 \mathrm{kHz}$. Deliver each run of 8 unipolar electric pulses in two trains of four pulses, with $1 \mathrm{sec}$ interval, in two perpendicular directions.

5. For needle electrodes with rows use pulse parameters: pulse duration $100 \mu \mathrm{sec}$, amplitude to electrode distance ratio $1,000 \mathrm{~V} / \mathrm{cm}$, frequency 1 or $5 \mathrm{kHz}$. Each run of unipolar electric pulses consists of eight pulses.

6. For needle electrodes, arrange in a hexagonal configuration and use pulse parameters: pulse duration $100 \mu \mathrm{sec}$; the hexagonal electrode has a fixed voltage of $730 \mathrm{~V}$ (if the distance between the electrodes is $7 \mathrm{~mm} ; 1,040 \mathrm{~V} / \mathrm{cm}$ ), with a frequency of $5 \mathrm{kHz}$ and the number of pulses depends on the number of needles. In case of 7 needles 24 pulses are applied

10. Disposal of contaminated material.

1. Dispose of all contaminated materials (syringes, needles, vials, gauzes, gloves) in a container intended for incineration.

\section{Follow-up}

NOTE: At each visit, tumor nodules are measured with a Vernier caliper and photographed. These visits also serve in order to evaluate the possible local and systemic side effects. Response to the treatment is scored after 4 weeks based on the criteria of "Response evaluation criteria in solid tumors" (RECIST) ${ }^{19}$ as a complete response (CR) - disappearance of the nodule, partial response (PR) - at least a $30 \%$ decrease in the sum of the diameters of the target lesion, taking as a reference the baseline sum diameters, progressive disease (PD) - at least a 20\% increase in the sum of the diameters of the target lesions, taking as a reference the smallest sum being studied, stable disease (SD) - neither a sufficient shrinkage to qualify for PR, nor a sufficient increase to qualify for PD, taken as a reference the smallest sum diameters. For all response definitions a minimum 4-week duration is required for qualifying each type of response. Observation time is calculated as the interval between the date of the first treatment and the date of the last examination of the patient. Possible side effects are determined according to the Veterinary Cooperative Oncology Group toxicity scale (VCOG - CTCAE) $)^{20}$ at each follow-up examination.

1. Schedule an appointment 1 week after the therapy. Schedule an appointment 2 weeks after the therapy. Schedule an appointment 4 weeks after the therapy. After 4 weeks schedule an appointment once a month.

\section{Representative Results}

The case presents a mast cell tumor located on the eyelid of a dog. The patient was treated with intratumorally-injected cisplatin and the application of 8 electric pulses using plate electrodes.

The size of the tumor was $5 \mathrm{~mm}$. Due to the sensitive area of the tumor's location general anesthesia was used. According to the size of the tumor $0.5 \mathrm{ml}(0.5 \mathrm{mg})$ of cisplatin was injected intratumorally. One min after the injection electric pulses were applied using an $8 \mathrm{~mm}$ plate electrode. Only one run of electric pulses was applied. After treatment the patient was supervised until it regained sufficient consciousness. The patient was released after two hours. After one week the patient came for a regular check-up. The tumor ulcerated and the area around the tumor was slightly swollen. The tumor was measured by Vernier caliper and photographed. One month after the treatment no further evidence of the tumor was noted. The wound was closed and cicatrix with alopecia was seen at the treatment site. After treatment the dog did not show any signs of pain, the function of the eyelid has been preserved during the entire healing period and the cosmetic effects after treatment are excellent. 


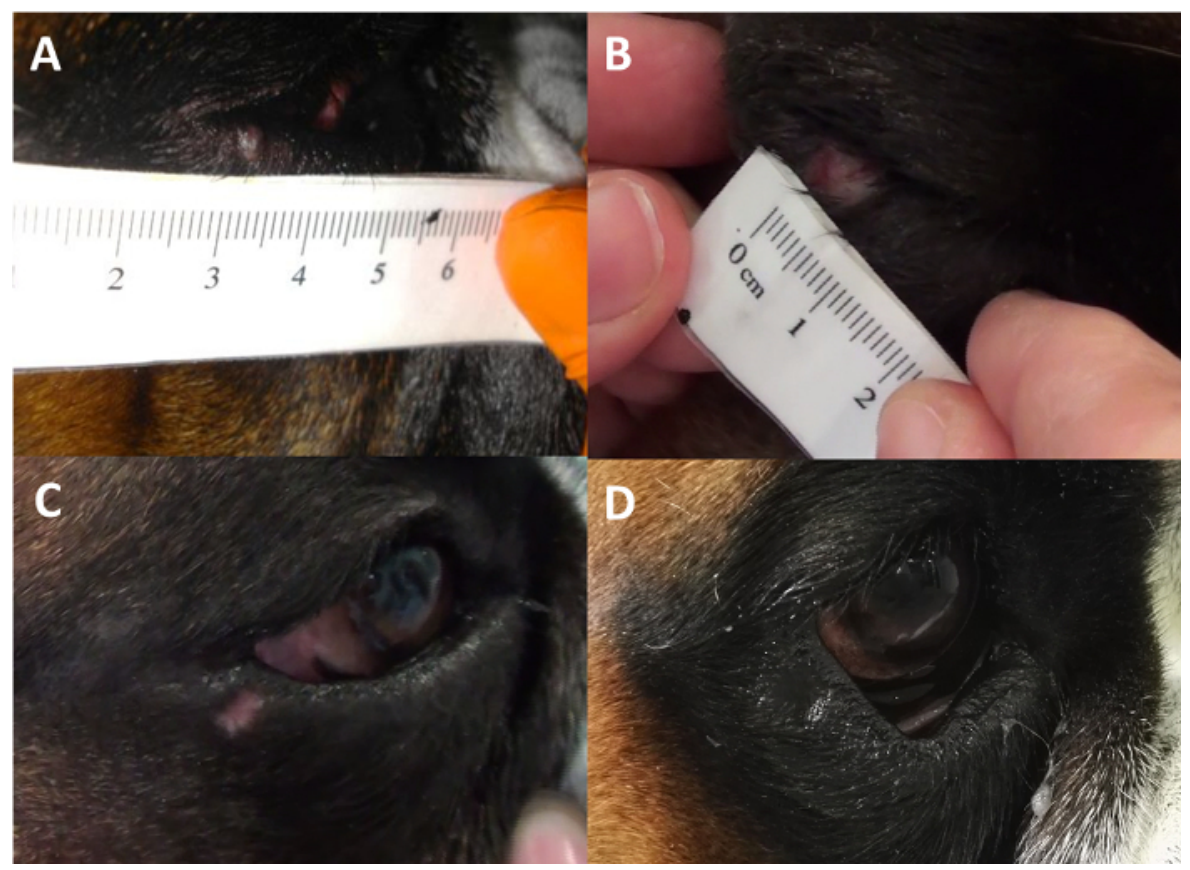

Figure 1. Electrochemotherapy treatment. The figure showing the dog before treatment (A), one week (B), one month (C) and three months after treatment (D). (A) Mast cell tumor on the lower eyelid. The 2.5-year old German boxer dog with a tumor size of $5 \mathrm{~mm}$ prior to the therapy. (B) After the therapy the tumor started to ulcerate and the tissue surrounding the electroporated area became slightly swollen. Two weeks after the therapy a crust formed at the tumor site and fell off (not shown). (C) One month after electrochemotherapy no evidence of the tumor was noted and cicatrix with alopecia was seen at the tumor site. (D) Three months after electrochemotherapy total re-epithelization occurred at the site of the tumor. Please click here to view a larger version of this figure.

\begin{tabular}{|l|l|l|}
\hline & Operating modality (OM) & B \\
\hline Number, volume and location of tumor nodules & A & \\
\hline $1-3$ nodules $<1 \mathrm{~cm}^{3}$ skin and oral tumors & $\sqrt{ }$ & $\sqrt{ }$ \\
\hline $\begin{array}{l}>3 \text { nodules, any volume skin and oral tumors } \\
\text { head }\end{array}$ & & $\sqrt{ }$ \\
\hline $\begin{array}{l}\text { any number, any volume of skin and oral } \\
\text { tumors head }\end{array}$ & & \\
\hline
\end{tabular}

Table 1. Selection of Operating modality is based on the number of tumor nodules and their size. Deep sedation = OM A; General anesthesia $=$ OM B.

\begin{tabular}{|l|l|l|}
\hline Chemotherapeutic drug/size of tumor & Intratumoral application & Intravenous application \\
\hline Cisplatin dose $(1 \mathrm{mg} / \mathrm{ml})<1 \mathrm{~cm}^{3}$ & $1 \mathrm{ml}(1 \mathrm{mg}) / \mathrm{cm}^{3}$ of tumor & \\
\hline Cisplatin dose $(1 \mathrm{mg} / \mathrm{ml})>1 \mathrm{~cm}^{3}$ & $0.5-1 \mathrm{ml}(0.5-1 \mathrm{mg}) / \mathrm{cm}^{3}$ of tumor & \\
\hline Bleomycin dose $(3,000 \mathrm{IU} / \mathrm{ml})<1 \mathrm{~cm}^{3}$ & $0.5 \mathrm{ml}(1,500 \mathrm{IU}) / \mathrm{cm}^{3}$ of tumor & $300 \mathrm{IU} / \mathrm{kg}$ \\
\hline Bleomycin dose $(3,000 \mathrm{IU} / \mathrm{ml})>1 \mathrm{~cm}^{3}$ & $0.5-1 \mathrm{ml}(1,500-3,000 \mathrm{IU}) / \mathrm{cm}^{3}$ of tumor & $300 \mathrm{IU} / \mathrm{kg}$ \\
\hline
\end{tabular}

Table 2. The appropriate injection volume of cytostatics according to the administration route and tumor size in dogs.

\begin{tabular}{|l|l|l|}
\hline Chemotherapeutic drug/size of tumor & Intratumoral application & Intravenous application \\
\hline Bleomycin dose $(3,000 \mathrm{IU} / \mathrm{ml})<1 \mathrm{~cm}^{3}$ & $0.5 \mathrm{ml}(1,500 \mathrm{IU}) / \mathrm{cm}^{3}$ of tumor & $300 \mathrm{IU} / \mathrm{kg}$ \\
\hline Bleomycin dose $(3,000 \mathrm{IU} / \mathrm{ml})>1 \mathrm{~cm}^{3}$ & $0.5-1 \mathrm{ml}(1,500-3,000 \mathrm{IU}) / \mathrm{cm}^{3}$ of tumor & $300 \mathrm{IU} / \mathrm{kg}$ \\
\hline
\end{tabular}

Table 3. The appropriate injection volume of cytostatics according to the tumor size in cats.

\section{Discussion}

Electrochemotherapy has proved to be highly effective against different primary tumors or metastases in dogs and cats. Electrochemotherapy can be used with curative intent for solitary or multiple cutaneous or subcutaneous tumor nodules or as an adjuvant treatment to surgery. The standard treatment of choice for several tumors in dogs, including mast cell tumors, is surgical excision of the tumor. A study comparing surgery 
as a standard treatment to ECT in treating mast cell tumors in dogs showed that treatment with ECT resulted in $70 \%$ of complete responses while surgery resulted in $50 \%$ of complete responses ${ }^{11}$ demonstrating that ECT is an equivalent treatment to surgery.

For the pronounced antitumor effectiveness of ECT two conditions have to be fulfilled, which are critical steps within the protocol. The first one is the administration of cytostatics. The presence of the drug in the target tissues determines ECT efficiency as the drug needs to be present inside the cells in order to kill them. When injecting the drug intratumorally, the sign of good tissue retention is tissue whitening. Also, some leakage of solution after administration could occur through the holes produced by the needle in the tumor or in necrotizing tissues during further ECT sessions. Thus, reaching $1 \mathrm{mg} / \mathrm{cm}^{3}$ (cisplatin) or $3,000 \mathrm{Ul} / \mathrm{cm}^{3}$ (bleomycin) concentrations could then be purely theoretical in those cases, however, the goal is to try to retain as much solution as possible in the target tissues. In addition, electric pulses should be applied as quickly as possible in order to obtain a vasoconstricting effect which contributes to solution retention.

Two different routes of administration can be used with ECT: intratumoral and intravenous. The antitumor effectiveness of ECT with intratumoral cisplatin or bleomycin or with intravenous bleomycin is comparable ${ }^{21}$ whereas electrochemotherapy with the intravenous application of cisplatin is less effective ${ }^{22}$ therefore cisplatin is only used in intratumoral administration. Another important feature of the cisplatin drug is that its use in cats is contraindicated as cisplatin has been shown to be highly toxic in cats ${ }^{23}$. If the patient has one or only a few nodules, then an intratumoral route is recommended. If the number of nodules exceeds $\sim 5$ nodules, then an intravenous route is recommended. Intratumoral injection of cisplatin has some antitumor effectiveness even without the application of electric pulses, while bleomycin, due to its high hydrophilic nature, does not. Therefore, cisplatin is the first drug of choice if intratumoral application is possible in dogs. This is important because of the second condition that must be fulfilled for the effective antitumor action of ECT and that is coverage of the whole tumor with a sufficiently high electric field. To obtain the increase in cell membrane permeabilization, the induced electric field within the tissue must exceed a certain threshold. The distribution of the electric field in the tissue depends on the electrodes, therefore it is important what kind of electrodes are used. Plate or parallel array electrodes can be safely used for small superficial tumors ${ }^{24}$. If the tumors are thicker and bigger, hexagonal electrodes are more suitable. An important fact is that the application of the electric pulses can be repeated, so that the whole tumor area is covered, including the safety margins. Since electric pulses cause vascular lock, their application should start from the edge of the tumor, towards its center.

Currently, the limitation of the technique is the availability of the electrodes which prevents the treatment of deep-seated tumors. However, the configuration of the electrodes is a technological feature that is fast developing and now different sets of electrodes are used for specific administrations long-needle single electrodes for metastases of colorectal tumors in the liver ${ }^{25}$, bone metastases ${ }^{7}$, soft tissue sarcoma ${ }^{26}$, endoluminal electrodes for colorectal, gastric and esophageal tumors ${ }^{27}$ and expandable "umbrella" type electrodes for brain tumors ${ }^{8}$.

ECT can be effective as a one-time treatment only, but in the case of failure or partial tumor response it can be repeated several times with improved effectiveness. ECT is a local ablative treatment. Its local effectiveness is up to $80 \%$ of local tumor control but without a noticeable effect on distant metastasis ${ }^{7}$, which is another limitation of the therapy. Therefore, to achieve a systemic response, ECT could be combined with other therapies. Recently, we proposed combining ECT with another biomedical application of electroporation, i.e., gene electrotransfer of the immunomodulatory molecule interleukin-12 (IL-12), which is known for its antitumor activity. Combination of ECT and gene electrotransfer with IL-12 has already been shown to be a safe combined therapy with no side effects and good local tumor with high complete response rate ${ }^{28}$, which also prevented recurrence of distant metastases.

In conclusion, the advantages of electrochemotherapy are its simplicity, short duration of treatment sessions, low cytostatic doses and insignificant side effects. In addition, it is performed on an outpatient basis, therefore, the patients do not have to stay in the hospital.

Nowadays, the availability of different generators of electric pulses on the market is increasing, leading also to the lowering of the price of the generators with no loss in reliability and thereby making electrochemotherapy even more affordable. These should all lead to the wider use of this therapy in veterinary medicine.

\section{Disclosures}

The authors have nothing to disclose.

\section{Acknowledgements}

The authors acknowledge the financial support received from the state budget by the Slovenian Research Agency (program no. P3-0003, project no. J3-6796). The research was conducted in the scope of the EBAM European Associated Laboratory (LEA). This manuscript is the result of the networking efforts of the COST Action TD1104. We wish to thank Alenka Seliskar, Mojca Juvan (Veterinary Faculty, Clinic for Surgery and Small Animals) and Jakob Konda (Institute of Oncology, Ljubljana, Ljubljana, Slovenia) for all of their valuable help.

\section{References}

1. Mir, L.M., Orlowski, S., Belehradek, J.Jr., \& Paoletti, C. Electrochemotherapy potentiation of antitumour effect of bleomycin by local electric pulses. Eur J Cancer. 27 (1), 68-72 (1991).

2. Sersa, G., Cemazar, M., \& Miklavcic, D. Antitumor effectiveness of electrochemotherapy with cis-diamminedichloroplatinum(II) in mice. Cancer Res. 55 (15), 3450-3455 (1995).

3. Sersa, G. et al. Electrochemotherapy in treatment of tumours. Eur J Surg Oncol. 34 (2), 232-240 (2008).

4. Sersa, G., Cemazar, M., Miklavcic, D., \& Chaplin, D. J. Tumor blood flow modifying effect of electrochemotherapy with bleomycin. Anticancer Res. 19 (5B), 4017-4022 (1999).

5. Markelc, B., Sersa, G., \& Cemazar, M. Differential mechanisms associated with vascular disrupting action of electrochemotherapy: intravital microscopy on the level of single normal and tumor blood vessels. PLoS One. 8 (3), e59557 (2013). 
6. Bellard, E. et al. Intravital microscopy at the single vessel level brings new insights of vascular modification mechanisms induced by electropermeabilization. J Control Release. 163 (3), 396-403 (2012).

7. Sersa, G. et al. Electrochemotherapy of tumors as in situ vaccination boosted by immunogene electrotransfer. Cancer Immunol Immunother 64 (10), 1315-1327 (2015).

8. Linnert, M., Iversen, H. K., \& Gehl, J. Multiple brain metastases - current management and perspectives for treatment with electrochemotherapy. Radiol Oncol. 46 (4), 271-278 (2012).

9. Bianchi, G., Campanacci L., Donati D. Electrochemotherapy in bone metastases: results of a phase II study. 7th Conference on Experimental and Translational Oncology.'Association of Radiology and Oncology. Portorož, Slovenia, 46-47, (2013).

10. Edhemovic, I. et al. Intraoperative electrochemotherapy of colorectal liver metastases. J Surg Oncol. 110 (3), $320-327$ (2014).

11. Kodre, V. et al. Electrochemotherapy compared to surgery for treatment of canine mast cell tumours. In Vivo. 23 (1), 55-62 (2009).

12. Spugnini, E. P. et al. Electrochemotherapy for the treatment of recurring aponeurotic fibromatosis in a dog. Can Vet J. 54 (6), 606-609, (2013).

13. Tozon, N., Pavlin, D., Sersa, G., Dolinsek, T., \& Cemazar, M. Electrochemotherapy with intravenous bleomycin injection: an observational study in superficial squamous cell carcinoma in cats. J Feline Med Surg. 16 (4), 291-299 (2014).

14. Tamzali, Y. et al. Successful treatment of equine sarcoids with cisplatin electrochemotherapy: a retrospective study of 48 cases. Equine Vet $J$. 44 (2), 214-220 (2012).

15. Mali, B. et al. Tumor size and effectiveness of electrochemotherapy. Radiol Oncol. 47 (1), 32-41 (2013)

16. Blackwood, L., et al. European consensus document on mast cell tumors in dogs and cats. Vet Comp Oncol. 10 (3), e1 - e29 (2012).

17. Ivorra, A., Al-Sakere B., Rubinsky B., Mir L.M. Use of conductive gels for electric field homogenization increases the antitumor efficacy of electroporation therapies. Phys Med Biol. 53 (22), 6605-18 (2008).

18. Suzuki, D.O.H., Marques, C.M.G., Rangel, M.M.M. Conductive Gel Increases the Small Tumor Treatment With Electrochemotherapy Using Needle Electrodes. Artif Organs. (2016).

19. Eisenhauer, E. A. et al. New response evaluation criteria in solid tumours: revised RECIST guideline (version 1.1). Eur J Cancer. 45 (2), 228-247 (2009).

20. Veterinary Co-operative Oncology Group - Common Terminology Criteria for Adverse Events (VCOG-CTCAE) following chemotherapy or biological antineoplastic therapy in dogs and cats v1.0. Veterinary and Comparative Oncology. 2 (4), 195-213 (2004).

21. Cemazar, M. et al. Electrochemotherapy in veterinary oncology. J Vet Intern Med. 22 (4), 826-831 (2008).

22. Sersa, G. Electrochemotherapy: animal model work review. Methods Mol Med. 37 119-136 (2000).

23. Knapp, D. W., Richardson, R. C., DeNicola, D. B., Long, G. G., \& Blevins, W. E. Cisplatin toxicity in cats. J Vet Intern Med. 1 (1), $29-35$ (1987).

24. Cemazar, M., Golzio, M., Sersa, G., Rols, M. P., \& Teissie, J. Electrically-assisted nucleic acid delivery to tissues in vivo: where do we stand? Curr Pharm Des. 12 (29), 3817-3825 (2006).

25. Edhemovic, I., et al. Electrochemotherapy: a new technological approach in the treatment of metastases in the liver. Technol Cancer Res Treat. 10 (5), 475-485 (2011).

26. Campana, L. G., et al. Electrochemotherapy treatment of locally advanced and metastatic soft tissue sarcomas: results of a non-comparative phase II study. World J Surg. 38 (4), 813-822 (2014).

27. Soden, D. M., et al. Successful application of targeted electrochemotherapy using novel flexible electrodes and low dose bleomycin to solid tumours. Cancer Lett. 232 (2), 300-310 (2006).

28. Cemazar, M., Ambrozic Avgustin, J., Pavlin, D., Sersa, G., Poli, A., Krhac Levacic, A., Tesic, N., Lampreht Tratar, U., Rak, M., Tozon, N. Efficacy and safety of electrochemotherapy combined with peritumoral IL-12 gene electrotransfer of canine mast cell tumours. Vet Comp Oncol. (2016). 